\title{
Control of carbon nanotube morphology by change of applied bias field during growth
}

\author{
L.-H. Chen, J. F. AuBuchon, A. Gapin, C. Daraio, P. Bandaru, and S. Jin ${ }^{\text {a) }}$ \\ University of California at San Diego, La Jolla, California 92093-0411 \\ D. W. Kim ${ }^{\text {b) }}$ and I. K. Yoo \\ Samsung Advanced Institute of Technology, PO Box 111, Suwon 440-600, Korea \\ C. M. Wang \\ Pacific Northwest National Laboratory, PO Box 999, Richland, Washington 99352
}

(Received 8 July 2004; accepted 21 October 2004)

\begin{abstract}
Carbon nanotube morphology has been engineered via simple control of applied voltage during dc plasma chemical vapor deposition growth. Below a critical applied voltage, a nanotube configuration of vertically aligned tubes with a constant diameter is obtained. Above the critical voltage, a nanocone-type configuration is obtained. The strongly field-dependent transition in morphology is attributed primarily to the plasma etching and decrease in the size of nanotube-nucleating catalyst particles. A two-step control of applied voltage allows a creation of dual-structured nanotube morphology consisting of a broad base nanocone ( $\sim 200 \mathrm{~nm}$ dia.) with a small diameter nanotube $(\sim 7 \mathrm{~nm})$ vertically emanating from the apex of the nanocone, which may be useful for atomic force microscopy. (c) 2004 American Institute of Physics.
\end{abstract}

[DOI: $10.1063 / 1.1830081]$

Carbon nanotubes (CNTs) have attracted much attention in recent years because of their unique electrical, mechanical, and various physical properties. ${ }^{1-8}$ For many potential applications, aligned CNT geometry is important. It is well known that vertically aligned nanotube arrays can be grown by plasma-enhanced chemical vapor deposition (PECVD) processing, either microwave ${ }^{9}$ or dc plasma. ${ }^{10-12}$

The growth of CNTs by dc PECVD involves many processing parameters, such as bias field, plasma power, temperature, chamber pressure, and feed gas composition composition. The growth direction of the nanotubes can be controlled by the electric field related to either applied bias or plasma-induced bias. In general, the CNTs synthesized by dc plasma CVD processing are of "nanofiber"-type multiwalled nanotubes with the graphene layer walls positioned at some angles rather than having the walls parallel to the long axis. ${ }^{12}$ The nanotubes grown by microwave plasma CVD or thermal CVD tend to have the carbon walls parallel to the long axis. These nanotubes with inclined walls are still denoted as "nanotubes" in this letter in a broad sense, as many of them still have hollow cores in the middle.

CNTs tend to grow in the electric-field direction, which is often perpendicular to the substrate surface. By controlling the applied bias, CNTs can grow in varied directions including the in-plane direction. ${ }^{13}$ More recently, multiple sharp bending of CNTs to produce a zig-zag morphology has also been demonstrated by repeatedly altering the applied field directions. ${ }^{14}$

While there have been a very large number of publications on nanotube growth itself, there have been few reports on enabling discoveries as to how to manipulate them to move up to the next level of scientific or technical advances. Engineering the shape of CNTs is an important issue for

\footnotetext{
a) Author to whom correspondence should be addressed; electronic mail: jin@ucsd.edu

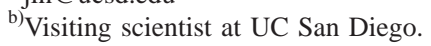

successful applications of nanotubes. A cone-shaped structure can provide mechanical stability yet provide very sharp tips, which may be useful for enhanced electron field emission. In the present work, we report how a manipulation of applied dc bias can be utilized to control the CNT morphology, from tube to cone shape, as well as to synthesize composite nanotube configurations during CVD growth.

Arrays of aligned CNTs with different morphologies were grown on Ni catalyst particles using a dc PECVD process. A nickel catalyst layer ( $\sim 5 \mathrm{~nm}$ thick) was first deposited onto the $n$-type $\mathrm{Si}$ (100) substrates. The base pressure of vacuum chamber was maintained at $\sim 5 \times 10^{-2}$ Torr of $\mathrm{H}_{2}$. Upon heating to $\sim 700{ }^{\circ} \mathrm{C}$, the $\mathrm{Ni}$ film broke up into islands with average diameters of $30-40 \mathrm{~nm}$. The ammonia gas $\left(\mathrm{NH}_{3}\right)$ was then fed into the chamber and a de bias of 450 , 500,550 , or $600 \mathrm{~V}$ was applied between the anode above the sample and the cathode just below the sample $(1 \mathrm{~cm}$ gap) to create the plasma. Because of the presence of plasma directly above the sample, the actual electric field that the nanotubes encounter can be significantly higher than the nominal field. Under the applied voltage, plasma formed and acetylene gas $\left(\mathrm{C}_{2} \mathrm{H}_{2}\right)$ was added to the chamber flowing at $30 \mathrm{sccm}$ with the total $\mathrm{NH}_{3}$ and $\mathrm{C}_{2} \mathrm{H}_{2}$ pressure at $\sim 3$ Torr. After $\sim 20 \mathrm{~min}$ in the plasma, $\sim 1-2-\mu \mathrm{m}$-long multiwalled nanotubes were formed.

Shown in Figs. 1(a)-1(d) are the SEM images of CNT samples grown on $\mathrm{Ni}(\sim 5 \mathrm{~nm}$ thick)-coated $\mathrm{Si}$ substrate at a dc bias voltage of 450, 500, 550, and $600 \mathrm{~V}$, respectively, for $20 \mathrm{~min}$. As the plasma power was kept in a small range of 35-50 W for different applied bias voltage, samples in this study were grown at similar temperatures. All other processing parameters, including the heating time and ammonia/ acetylene gas composition, were kept constant. The striking difference in CNT morphology induced by the voltage change is apparent from the figure. At the lower bias fields, such as 450 and $500 \mathrm{~V}$, slender, straight, and equidiameter nanotubes $\sim 30-60 \mathrm{~nm}$ in diameter were grown vertically 

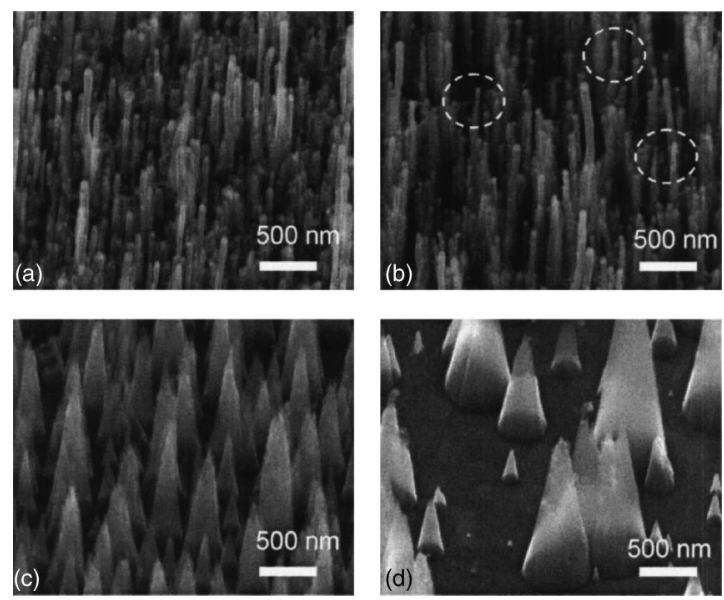

FIG. 1. SEM images of CNT samples grown on Ni (5 nm)-coated Si substrate at a dc bias of (a) $450 \mathrm{~V}$, (b) $500 \mathrm{~V}$, (c) $550 \mathrm{~V}$, and (d) $600 \mathrm{~V}$ for 20 $\min$.

aligned, as shown in Figs. 1(a) and 1(b). The Ni catalyst particles with about the same diameters as those for the nanotubes are present on top of all nanotubes, indicating the occurrence of tip-growth mechanism. In contrast to the 450 $\mathrm{V}$ sample, the $500 \mathrm{~V}$ sample [Fig. 1(b)] begins to show the tendency toward transition to a cone-like structure, with the nanotube diameter still wire-like but becoming slightly tapered and getting larger at the lower part of the nanotubes. It is also noticeable that the catalyst particle diameter is beginning to get smaller as compared to the $450 \mathrm{~V}$ sample.

For a higher applied voltage of $550 \mathrm{~V}$ [Fig. 1(c)], predominantly nanocone-like nanotubes with very sharp tips are obtained. The base diameter at the bottom of nanocones is much larger than the nanotube diameter of Fig. 1(c), with the cone base diameter varying from 100 to $300 \mathrm{~nm}$. It is important to note that almost all nanocones show no Ni catalyst particle on the tip, which indicates that the carbon nanotubes essentially stopped growing once the nanocone structure is completed. Perhaps related to this is the observation that the nanocone tips are very sharp, with the radius of curvature estimated to be as small as $\sim 5 \mathrm{~nm}$ in diameter. At an even higher bias of $600 \mathrm{~V}$ [Fig. 1(d)], the nanocones become much shorter and the aspect ratio gets smaller. These cones have larger base diameters as large as $500 \mathrm{~nm}$, and their density is much lower compared to the ones grown at $550 \mathrm{~V}$.

TEM images of the samples grown at 450 and $550 \mathrm{~V}$, respectively, for $20 \mathrm{~min}$, are shown in Figs. 2(a) and 2(b). Figure 2(a) shows the tube-like structure with a $\mathrm{Ni}$ catalyst cap on top of CNTs. Figure 2(b) is the TEM micrograph of a broken-off nanocone from the $550 \mathrm{~V}$ sample. The cone is crystalline with some inclusions. In some of the nanocones

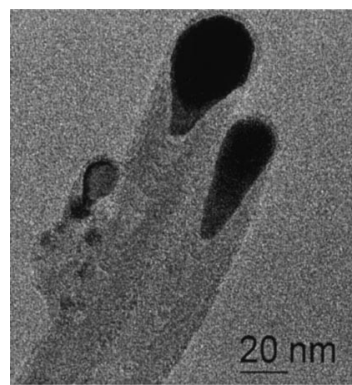

(a)

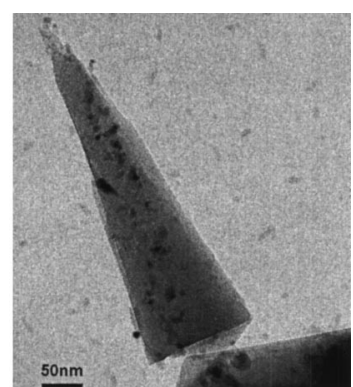

(b)
FIG. 2. TEM images of CNTs grown at (a) $450 \mathrm{~V}$ and (b) $550 \mathrm{~V}$ for 20 min.
Downloaded 28 Sep 2006 to 131.215 .225 .9 . Redistribution subject

FIG. 2. TEM images of CNTs grown at (a) $450 \mathrm{~V}$ and (b) $550 \mathrm{~V}$ for 20 min.
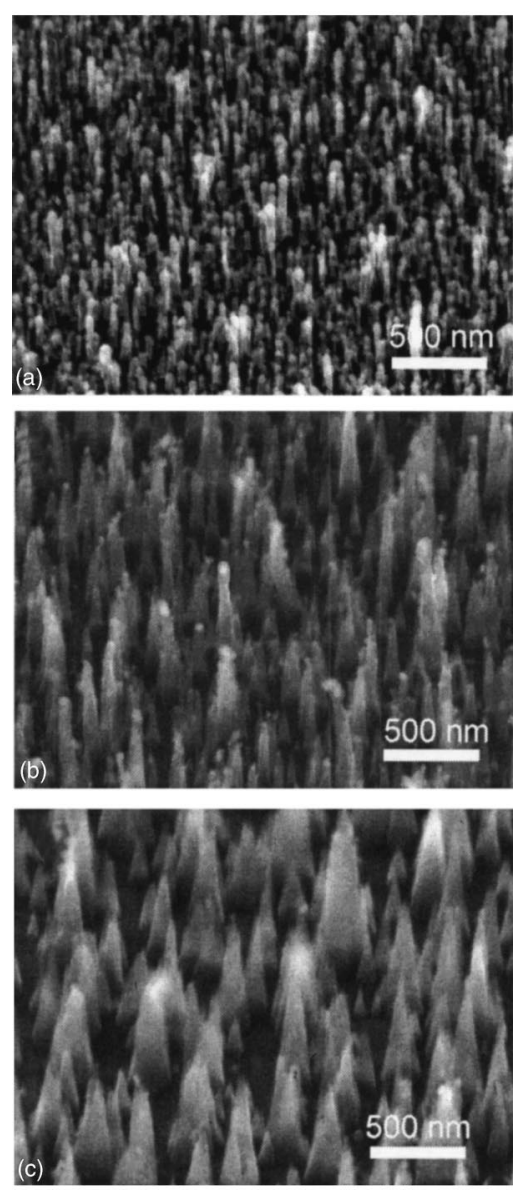

FIG. 3. Nanocone morphology vs CVD growth time at $550 \mathrm{~V}$ for (a) $2 \mathrm{~min}$, (b) $6 \mathrm{~min}$, and (c) $14 \mathrm{~min}$.

there appears to be a darker, shell-like region. EDX spot analysis indicates that the cone matrix contains carbon as well as Si. The tiny inclusions, which may be present either inside or on the surface of nanocones, were identified to be mainly composed of Ni. Further microstructural analysis is required to fully understand the detailed structure of the nanocones.

To better understand the formation process of the nanocone structure, we monitored the change of microstructure as a function of time during the CVD at a constant bias of 550 V. Figures 3(a)-3(c) display SEM microstructures of the samples CVD grown for 2, 6, and 14 min, respectively. As is evident from the figures, the CVD starts out with the formation of equidiameter nanotube structure on $\mathrm{Ni}$ catalyst particles, as shown in the 2 min sample of Fig. 3(a). On longer CVD for $6 \mathrm{~min}$, these nanotubes are quickly transformed to the nanocone structure, as shown in Fig. 3(b). The Ni catalyst particles begin to get smaller as compared to the $2 \mathrm{~min}$ sample. On a longer time CVD of 14 min [Fig. 3(c)], only nanocones are left, with their base diameter increasing with CVD time. On additional CVD (e.g., 20 - $30 \mathrm{~min}$ ), it is seen that the nanocone base diameter increases and the aspect ratio decreases.

The mechanism for the strong electric-field dependence of nanotube morphology observed in this work is described as follows. The formation and geometry of the nanotube versus nanocone structure appear to be dictated by the plasma etching of catalyst particles at the growth tip. During the dc plasma CVD, plasma etching of the Ni catalyst particles occurs continuously if the applied voltage (and hence the enAlP license or copyright, see http://apl.aip.org/apl/copyright.jsp 
ergy of plasma sputtering) is sufficient. SEM examinations of various samples clearly indicate that the size of catalyst particle on top of CNTs decreases as a function of time in the presence of plasma. The reduced catalyst size limits the kinetics of carbon uptake and nanotube growth along the upward direction, while the lateral growth continues with the continued addition of carbon from the CVD gas. Eventually, the vertical growth stops completely when the last small catalyst particle is sputtered away. The nanocones contained some $\mathrm{Si}$ as seen by our EDX analysis in TEM.

At a low bias voltage of $450 \mathrm{~V}$, the plasma energy is lower and the plasma etching effect (sputtering effect) is not strong enough to significantly reduce the size of the Ni catalyst particles. The lateral growth of nanocones is negligible and not obvious. Due to the continued existence of the $\mathrm{Ni}$ catalyst particles on the tip [Fig. 1(a)], longer nanotubes are obtained as the vertical growth lasts much longer. When the bias is raised to $500 \mathrm{~V}$ or higher, the plasma etching effect begins to reduce the particle size (e.g., see the nanotubes marked by circles) and hence the upward nanotube growth slows down and base lateral growth begins to become enhanced. The morphology of nanotubes begins to take the form of a slight nanocone shape. When the bias voltage is further raised to $550 \mathrm{~V}$, the magnitude of the catalyst sputter etching is increased to the extent that the Ni particles are completely sputtered away, and only the base lateral growth occurs [Fig. 1(c)]. At an even higher bias voltage of $600 \mathrm{~V}$, the nanocones just continue to have the lateral growth [Fig. 1(d)]. It appears that coarsening of the nanocone occurs at the expense of other nanocones.

The observed phenomenon of strong field dependence of nanotube morphology can be utilized to design and fabricate some unique shapes of nanotubes, for example, a nanotube configuration useful for conductance AFM tip. As is well known, the resolution of AFM imaging is determined by the sharpness, size, and shape of the probe tip. Typical commercially available AFM probe tips are made of silicon or silicon nitride $\left(\mathrm{Si}_{3} \mathrm{~N}_{4}\right)$ that is microfabricated into a pyramid configuration. Such probes exhibit a limited lateral resolution, and their rigid pyramidal shape does not allow easy access to narrow or deep structural features.

Utilizing the advances in carbon nanotube science and technology, an important development in probe technology might be achieved by attaching a small-diameter CNT on AFM tips. ${ }^{8}$ However, with the still relatively large nanotube dimensions utilized so far, potential improvements in lateral resolution were not seriously investigated. The long and slender geometry of CNTs (high aspect ratio) offers obvious advantages for probing narrow and deep features. The elastically compliant behavior of high aspect ratio nanotubes is also advantageous. While the attachment or growth of CNTs on AFM tips has been demonstrated, such nanotubes are not always straight and vertically positioned, and the adhesion strength, reproducibility/reliability in shape, size, and attachment angle of nanotube probes still remain as major issues.

Manipulating the applied electric field during CVD growth of nanocones, we have demonstrated a direct creation of dual-structured nanotube configuration. It consists of a broad and mechanically stable nanocone base $(200-500 \mathrm{~nm}$ base diameter) and one very thin and straight nanotube probe emanating vertically from the nanocone apex, as illustrated

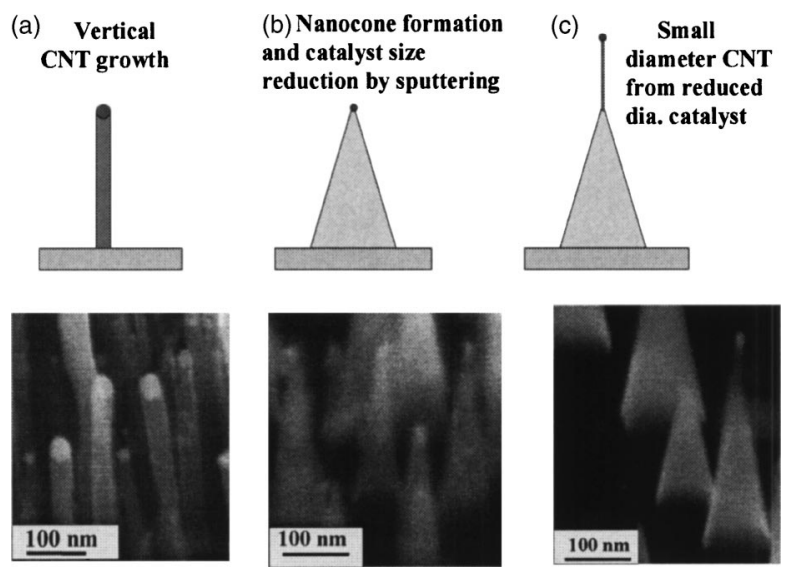

FIG. 4. Controlled nanotube tip geometry: (a) straight nanotube, (b) nanocone with small-diameter catalyst, and (c) dual-structured nanotubes synthesized by high-field and low-field two-stage CVD.

and supported by example SEM micrographs in Fig. 4. Instead of growing the standard, vertically aligned nanotube structure of Fig. 4(a) by low-field CVD, we start with growing a nanocone first, for example, using a higher bias voltage, such as 550-600 volts, or alternatively, by allowing a longer CVD time of 5-10 min as in the case of Fig. 3. Such a CVD treatment prepares a base nanocone structure and at the same time reduces the catalyst particle size to a few to several nanometers [Fig. 4(b)]. By intentionally lowering the applied voltage at this stage (e.g., to $450 \mathrm{~V}$ ), and continuing on with CVD, we nucleated and grew a single, straight nanotube from the apex of the broad nanocone utilizing the stillremaining, very small catalyst particles. The diameter of the vertically aligned nanotube on top of each of the nanocones is estimated to be $\sim 7 \mathrm{~nm}$, as shown in Fig. 4(c).

The authors acknowledge the support of the work by University of California Discovery Fund under Grant No. ele02-10133/Jin, NSF NIRTs under Grant Nos. DMI0210559 and DMI-0303790, and Lawrence Livermore National Laboratory under Grant No. MI-04-006.

${ }^{1}$ S. Iijima, Nature (London) 354, 56 (1991).

${ }^{2}$ C. Dekker, Phys. Today (5), 22 (May 1999).

${ }^{3}$ W. Zhu, C. Bower, O. Zhou, G. Kochanski, and S. Jin, Appl. Phys. Lett. 75, 873 (1999).

${ }^{4}$ C. Bower, W. Zhu, D. Shalom, D. Lopez, L. H. Chen, P. L. Gammel, and S. Jin, Appl. Phys. Lett. 80, 3820 (2002).

${ }^{5}$ A. Fennimore, T. Yuzvinsky, W. Han, M. Fuhrer, J. Cumings, and A. Zettl, Nature (London) 424, 408 (2003).

${ }^{6}$ S. Tans, A. Verschueren, and C. Decker, Nature (London) 393, 49 (1998).

${ }^{7}$ T. Rueckes, K. Kim, E. Joselevich, G. Tseng, C. Cheung, and C. Lieber, Science 289, 94 (2000).

${ }^{8}$ S. S. Wong, J. D. Harper, P. T. Lansbury, and C. M. Lieber, J. Am. Chem. Soc. 120, 603 (1998).

${ }^{9}$ C. Bower, W. Zhu, S. Jin, and O. Zhou, Appl. Phys. Lett. 77, 830, (2000).

${ }^{10}$ Z. F. Ren, Z. P. Huang, J. W. Xu, J. H. Wang, P. Bush, M. P. Siegal, and P. N. Provencio, Science 282, 1105 (1998).

${ }^{11}$ M. Chhowalla, K. B. K. Teo, C. Ducati, N. L. Rupesinghe, G. A. Amaratunga, A. C. Ferrari, D. Roy, J. Robertson, and W. I. Milne, Appl. Phys. Lett. 90, 5308 (2001).

${ }^{12}$ V. I. Merkulov, M. A. Guillorn, D. H. Lowndes, and M. L. Simpson, Appl. Phys. Lett. 79, 1178 (2001)

${ }^{13}$ C. M. Hsu, C. H. Lin, H. L. Chang, and C. T. Kuo, Thin Solid Films 420-421, 225 (2002).

${ }^{14}$ J. F. AuBuchon, L.-H. Chen, C. Daraio, and S. Jin, Nano Lett. 4, 1781 (2004). 